\title{
DISERTACIÓN ACERCA DEL BIEN MORAL EN LA EDUCACIÓN Y FORMA DE LLEVARLO A LA ESCUELA
} (Essay about the moral good in education
and how to implement it at the school)

Por Liliana Robledo*

Palabras Clave:

Escuela, moral, pedagogía, educación, auto realización, valores, ética, civica

\section{Abstract}

When it is inquired on the last scope of education, a number of questions arise with reference to what it is currently offered as education in the Colomblan educational institutions. The aim of our education is knowledge, being its topics so extensive and deep that they are barely permeated. This concern for offering quality knowledge is plausible. Nevertheless, the scope of knowledge has been fading away. This scope deals with the true sense of education as the formation of the future citizen for the societal wellbeing, in the search of the moral good.

Cuando se consulta sobre el sentido último de la educación se abren numerosos interrogantes ante lo que se brinda hoy como educación

- Candidata a Ph.D en Ética, Licenciada en Blologia y Quimica por la Unlversidad de Caldas. 
en las instituciones educativas colombianas.

Nuestra educación tiene como fin el conocimiento y los temas de él son tan amplios y profundos que apenas se logra permear en ellos. Esa preocupación por brindar un conocimiento de calidad está bien. Sin embargo se ha ido perdiendo el para qué de esos conocimientos. Finalidad que tiene que ver con el verdadero sentido de la educación que es la preparación del futuro ciudadano en lo que es bueno para todos, en la búsqueda del bien moral.

Así está expresado desde La Declaración Universal de Derechos Humanos. En su parágrafo 26.2. dice asi:
La educación tendrá por objeto el pleno desarrolio de la personalidad humana y el fortalecimiento del respeto a los derechos del hombre $y$ a las libertades fundamentales; favorecerá la comprensión, la tolerancia y la amistad entre todas las naciones y todos los grupos étnico religiosos, y promoverá el desarrollo de las actividades de las Naciones Unidas para el mantenimiento de la paz.

Cualquiera que lea el texto puede estar de acuerdo con él, pero se abren muchos interrogantes, por ejemplo: ¿qué tiene que ver el contenido del texto con la vida cotidiana de la institución educativa?, ¿qué es eso del pleno desarrollo de la personalidad?, ¿en qué momento se fortalece en la escuela el respeto por los derechos del hombre?...

Es alrededor de estos cuestionamientos que este articulo pretende argumentar, sin que por ello queden ellos exhaustivamente respondidos. Se trata mas bien de abrir la polémica y aportar algunos argumentos.

Se trabajarán cuatro temáticas que pretenden constituirse en los argumentos sobre los que se alimentarian las respuestas a los interrogantes hechos anteriormente: 1. El bien moral en la 
educación; 2. Diferencla entre una educación para el bienestar de una educación para la autorrealización; 3. Aspectos que dificultan que la pedagogía esté al servicio del bien moral; 4. Necesidad de involucrar los valores de una ética civica en la institución escolar, tanto al nivel del currículo como en la evaluación, e igualmente en la administración del sistema educativo.

\section{El bien moral en la educación}

El primer tema, que en un principio pareció ser el más fácll, se convirtió en un tema bastante extenso pues el bien moral se debió consultar con la tradición, tal vez ella fuera la que brindaría una luz acerca del desfase con la realidad que se manifiesta en la lectura del texto en referencia. Cortina dice, parafraseando a Rawls, refiriéndose a la consulta con la tradición:

La tarea del filósofo consistirá entonces- cree Rawls- en ayudarnos a comprender mejor lo que ya compartimos, y propone con tal fin rastrear en nuestras tradiciones cuál o cuáles darán mejor razón de ello, para pasar después a configurar conceptualmente con su ayuda un modelo que pueda devolverse a la sociedad para llevar a cabo la "tarea social práctica" de reforzar sus convicciones morales (1).

Al seguir a Rawls y estudiar la tradición desde los argumentos que a continuación se describen, se concluye que el bien moral de la educación se habia ido transformando y haciendo más complejo y extenso en las diferentes etapas de la vida de los pueblos de occidente, hasta confundirse con los requerimientos de la razón instrumental, en la educación actual.

Veamos: en la antigua Grecia el bien moral o la virtud se inspiraba en el héroe de los relatos homéricos. Marrou (2), describe la ética homérica como una moral heroica del honor, plantea que el eje de esa ética aristocrática fue el amor a la gloria. El héroe homérico no aicanza la felicidad si no se valora a si mismo, si no se afirma como el
1 Cortina. Adela, Ética aplicada y democracia radical, Tecnos, Madrid, 1997, p, 207. 2 Marrou. Henry-Irenee. Historia de la educación en la antigüedod, Akal, Madrid, 1985, p, 28.

\section{Á N}

Universided Autónona de Marizales

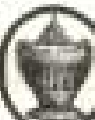


primero, distinto y superior, dentro de su categoria. En consecuencia, el ciudadano griego se cultivaba en esa visión de la virtud para servir a su pueblo. Así nos lo hace ver Sabine cuando toma de la oración fúnebre de Pericles el siguiente texto:

Cada uno de nosotros de cualquier estado o condición que sea, si tiene algún conocimiento de virtud, tan obligado está a procurar el bien y honra de la ciudad, como los otros, y no será nombrado para ningùn cargo, ni honrado, ni atacado por su linaje o solar, sino tan sólo por su virtud y bondad. Que por pobre y por bajo que sea con tal que pueda hacer bien y provecho a la República, no será excluido de los cargos y dignidades públicas(2).

El cultivo de la virtud se realizó por medio de la educación, llegándose a desarrollar todo un sistema formal de ella. La educación cultivaba el cuerpo y el espíritu. Lo primero por medio de los entrenadores deportivos y lo segundo desde el cultivo de las artes y los textos homéricos. El uso de los textos involucró a las letras en el sistema educativo. Ese sistema fue la clave para que la cuitura griega trascendiera sus fronteras y se extendiera a todo el mundo helénico y más tarde a Roma. Por otro lado, los oficios y el aprendizaje de esos oficios, no eran considerados dentro de las virtudes ciudadanas. Se concebia el aprendizaje de los quehaceres técnicos y cotidianos como algo ajeno a la educación, como un asunto propio de la servidumbre, algo que no le brindaba prestancia al ciudadano griego.

Roma heredó el pensamiento griego. Sin embargo, en ella, el cultivo de las letras y las artes tuvieron mayor preponderancia. También se transformó la virtud. El cludadano romano asumió como bien moral el cumplimiento de los deberes cívico-religiosos. El conocimiento vinculado a la idea de educación fue el de la filosofia, la retórica, la formación literaria y la oratoria. En éste se preparaban los cludadanos para el ejercicio de la politica, comprendida como el bien común y encarnada en el Estado.

También se desarrolló en el imperio romano todo un sistema formal
2 Sabine, George $H_{\text {, }}$ Historia de la teoría politica, Fondo de cultura económica, México, 1970 , p. 23.

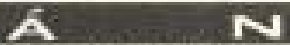


de educación, similar al griego. Todo ello a, udó a que se mantuviera y se hiciera fuerte el imperio. La existencia de una buena cantidad de ciudadanos educados, seguramente fue el puntal que facilitó la supervivencia de la moralidad y la civilidad cuando el imperio decayó. MacIntyre lo expresa de esta forma:

Se dio un giro crucial en la antigujedad cuando hombres y mujeres de buena voluntad abandonaron la idea de defender el imperium. En su lugar se pusieron a buscar, a menudo sin darse cuenta completamente de lo que estaban haciendo, la construeción de nuevas formas de comunidad dentro de las cuales pudiera continuar la vida moral de tal modo que moralidad y civilidad sobrevivieron a las épocas de barbarie y oscuridad que se avecinaban (3),

Por otro lado el sistema educativo formal que se llegó a desarrollar en el imperio romano se convirtió en la puerta de entrada del cristianismo, en dos sentidos: El primero tiene que ver con la semilla ética de los valores humanísticos, que ya había sido sembrada en el imperio romano por el estoicismo. El segundo, tiene que ver con la difusión de las sagradas escrituras. El alto grado de alfabetismo que se llegó a tener en el imperio romano, permitió que ellas se expandieran rápidamente en todo el mundo civilizado.

En la edad media el bien moral fue dificil de afianzar. Finalmente se consolidó alrededor de la revelación, al amparo de las interpretaciones que la jerarquía eclesiástica, bajo un conocimiento escolástico, aprobaba de las sagradas escrituras. El sistema educativo formal sólo tenia en cuenta a pocos privilegiados, que acogidos por el cuerpo eclesiástico, recibian una educación escolástica. En ésta época la educación continúa estando al servicio de la virtud.

La Modernidad que se inicia con el advenimiento de La Reforma, EI Renacimiento, La Ilustración, exigen una nueva forma y contenido a la educación. Ella debe atender a la formación moral y a la erudición. La educación, que hasta entonces sólo estaba al servicio del

3 Macintyre, Alastair, Tras la virtud, Critica, Barcelona, 2002, p. 322.

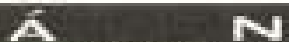

Uninersidad Auitónoma de Manizales
IF

Nha 11, Número 19, agosto - dickembre 2004 
aprendizaje de la doctrina cristiana, debe servir a nuevos paradigmas: el científico, el tecnológico y el económico. También se debe proyectar para atender un número mucho mayor de individuos, debe convertirse en la transmisora de las nuevas ideas politicas y debe capacitar a un gran número de individuos en los nuevos oficios. En conclusión se debe ampliar en cuanto a su fin último. En otras palabras, la educación se debe poner al servicio de todas las nuevas necesidades del ser humano, asunto que provoca que el fin de la educación de los antiguos -el cultivo de la virtud-, se vaya diluyendo hasta tener que plantearse la pregunta acerca de cuál es la virtud en la educación contemporánea.

De acuerdo con el texto emanado de la Declaración Universal de Derechos Humanos, la virtud de la educación contemporánea tiene que ver con el pleno desarrollo de la personalidad humana y el fortalecimiento de los derechos humanos. Ahora bien, contrario a la tendencia actual, el desarrollo de la personalidad humana involucra la autonomia y la autorrealización. Cortina expresa al respecto:

\footnotetext{
Deberiamos preguntarnos... si lo que queremos promocionar a través de la educación son sólo individuos técnica y socialmente diestros, que saben manejarse para lograr su bienestar, o personas autónomas con afán de autorrealización, porque- como sabemos- no es lo mismo el bienestar que la autorrealización. Para lograr el primero basta con las destrezas, para conseguir la segunda, es necesario una educación moral, en el más amplio sentido del teérmino "moral" (4).
}

Para fortalecer esa educación moral se debe luchar por el afianzamiento de los derechos humanos. Afianzamiento que debe sustentarse en los valores propios de ellos: la libertad, la igualdad, la solidaridad. Igualmente no pueden quedarse por fuera el diálogo y el respeto activo. El diálogo es la base para sustentar la autonomía del hombre moderno, al considerar el otro como un interlocutor válido, $y$

4 Cf. Cortina. Adela, Ética aplicada y democracia radical, Tecnos, Madrid, 1997, p, 214.
A N

Universidad Ausoboma de Manizales
E

Alo 11, Nimeio 19, agosto- cldembre 2004 
el respeto activo sustenta la dignidad que hace del hombre moderno un ser que no tiene precio. De nuevo Cortina nos brinda argumentos para ello, asi se expresa:

\begin{abstract}
es imposible construir una sociedad auténticamente democrática contando con individuos técnica y socialmente diestros, porque tal sociedad ha de sustentarse en valores para los que la razón instrumental es ciega, valores como la autonomia y la solidaridad, que componen de forma inevitable la conciencia racional de las instituciones democráticas (5).
\end{abstract}

En conclusión, la virtud de la educación actual tiene que ver, igualmente, con una educación para la autonomía y para la autorrealización. Es indudable que ésta última no se alcanza sin el desarrollo del conocimiento y la tecnología. Empero, ese desarrollo que es necesario, no es suficiente. Para alcanzar la suficiencia debe estar acompañado de un bien moral.

\title{
Diferencia entre una educación para el blenestar de una educación para la autorrealización
}

Para adentrarse en esta temática se deben analizar dos aspectos relevantes:

- La educación contemporánea tiene un fuerte énfasis en la instrucción.

- La realidad contemporánea le solicita a la educación que haga énfasis en formación moral o educación para la autonomía.

El primer aspecto se analiza desde la realidad de la institución escolar actual. Ella gira alrededor de un curriculo que se confunde con el llamado plan de estudio. Este último se atiende bajo un sistema de dosificación del tiempo, que reparte la totalidad del uso de él, en un sistema denominado horario. El horario se satura con asignaturas

5 Ibidem. p, 213.

\section{A N}

Universidad Autinems de Manizalcs 
que son atendidas por los docentes de acuerdo a un plan preestablecido e igual para todos los grados en que se parcela el plan de estudio. Los resultados se valoran alrededor de unas competencias mínimas de conocimiento, que serán sometidos a evaluaciones parciales y a un rasero final. Esta valoración clasifica a unos individuos como exitosos y descalifica a los otros. Por otro lado, esta clasificación afecta también a las instituciones. Igualmente, hay que reconocer que esa evaluación, convertida en una competencia por el conocimiento, no tiene en cuenta las diferencias individuaies, sociales y económicas de los participantes a lo largo del proceso de capacitación (insuficientemente llamado formación), ni aclara nada acerca de la altura moral a la que puedan haber llegado los mismos.

Con las deficiencias éticas ya anotadas en el campo de la educación, la sociedad elige a los estudiantes de éxito, brindándoles el acceso a la educación superior, para proyectar sobre ellos la responsabilidad del manejo político y económico de las instituciones de carácter público y de carácter privado del país.

El segundo aspecto -el de la necesidad de brindar una educación con énfasis en el desarrollo moral- es consecuencia de las deficiencias del primer aspecto ya planteado. Cuando se consulta la realidad social, política y económica del pais, se confirma que la deficiencia de la educación actual está en la educación para la autonomía, que es esencial para el pleno desarrollo de la personalidad humana.

Para comprender aquello del pleno desarrollo de la personalidad humana en los tiempos modernos, hay que remitirse al concepto de hombre de cuño kantiano que establece que el ser humano es un ser con dignidad, por ello no es comparable con nada y es la razón por la cual no se le puede poner precio. Por tanto, es un ser autónomo, con capacidad de razonar y de argumentar sin someterse a dogmas o principios revelados. Busca el bien moral bajo principios universalizables. Por tanto, el pleno desarrollo de la personalidad humana no se ciñe solamente al bienestar, ni al placer material, $\mathrm{ni}$ al ejercicio del poder que le puede brindar una educación centrada en la 
Instrucción. Se ciñe a la posibilidad de ejercer su autonomia, tanto en lo moral como en el conocimiento, en el marco de los valores de una ética de minimos, orientada a la convivencia de seres humanos de cualquier cultura, credo o condición social.

\section{Aspectos que dificultan que la pedagogía esté al servicio del bien moral}

Para determinar qué aspectos de la pedagogía se ponen al servicio del bien moral del hombre moderno, se hace necesario retomar los origenes de la pedagogía moderna y conocer al menos la esencia de algunas de las innovaciones educativas del siglo XX.

La pedagogía, que nace con Comenio y llega hasta hoy, si se coloca al servicio del bien moral, pues su pretensión ha ido encaminada a la autorrealización del hombre. Es en la forma de hacerlo en donde se enmaraña, pues ha debido responder a diferentes concepciones ético-morales. En otras palabras, cada escuela pedagógica desde Comenio hasta hoy, ha debido construirse desde una concepción de hombre. Tal concepción ha ido desde la idea de que él está hecho a imagen y semejanza de Dios hasta la de que él es un ser autónomo y con dignidad. Entre esas concepciones de hombre se han hecho planteamientos pedagógicos diferentes que atienden de una u otra forma los valores que se han ido consolidando en la modernidad y pretenden atender, asi sea en forma parcial, la educación para la autonomia. Cada uno de esos planteamientos se ha centrado en aspectos diferentes de la didáctica, sin embargo no se ha logrado ubicar los valores propios de una ética civica en la vida de la escuela.

Son varios los aspectos que en este sentido se deben resaltar:

- Tanto la pedagogía tradicional(6) como la pedagogía contemporánea (7) parten de un concepto de hombre. La tradicional, que se podria Iniciar desde Comenio, por ser él el primer pedagogo de la era moderna, asumia al hombre como alguien hecho a imagen y semejanza de Dios. La pedagogía contemporánea asume al hombre como alguien autónomo, es
6 Determinar bajo un autor o fecha exacta lo que hoy en dia se denomina pedagogia tradicional no es posible. $A$ ella se hace referencia cuando se pretende hablar de un antes, con una estructura rigida y autoritaria donde el centro de referencia era el maestro y el conocimiento que êl deseaba impartir.

7 Igualmente a como ocurre con la pedagogia tradicional, la pedagogía contemporánea no posee un autor especifico ni una fecha de inicio. Se habla de ella cuando se pretenden expresar ideas contrarias a autoritarismo propio de la pedagogía tradicional

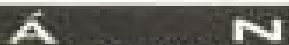

Universidad Nobhoma de Mancales 
decir como alguien con capacidad de razonar y de argumentar, bajo criterios universales.

- Las innovaciones pedagógicas han trabajado en función de una educación para la autonomía pero esas innovaciones pedagógicas se han asumido como instrumentos didácticos dentro de las instituciones educativas.

- Ninguna de las pedagogias, afortunadamente, ha planteado la posibilidad de clasificar al hombre de acuerdo con los niveles de instrucción alcanzados. Simplemente plantean la mejor forma de educar el hombre que hay en cada individuo.

- Las pedagogias están pensadas para estructurar los saberes y los presaberes en el individuo, asumiendo que él hace la búsqueda de ellos motivado por su propla búsqueda del bien moral, no bajo coacción o por la mera búsqueda del mejoramiento material, que son la base de la motivación de las instituciones educativas.

- Se confunden los medios con los fines. Se asume la educación para la autonomía como un aditamento de la instrucción. Igualmente tampoco se separan los valores morales de los valores materiales que la escuela atiende, tergiversándoșe as! la concepción de hombre que se pretenda atender, cayendo en el error de terminar objetivando al educando; y las acciones que apuntan hacia una educación para la autonomia se pierden.

- La pedagogia tradicional centra su forma de trabajo en el docente. Él, actúa como un planificador y dosificador del conocimiento. Conocimiento que se imparte en la clase magistral, haciendo del alumno un ser heterónomo. Las innovaciones pedagógicas han pretendido ser contestatarias ante esa forma de trabajo, buscando que el sujeto se estructure por si mismo, para responder de una manera autónoma.

- Los avances de la pedagogía, principalmente desde el arribo al 
siglo $\mathrm{XX}$, centran la forma de trabajar en el alumno. Sin embargo, se cae en el instrumentalismo, cuando se considera el rol del docente como el de un facilitador de material didáctico. Él, por el contrario, debe ser el representante, no sólo de la comunidad académica ante el estudiante, sino también el de la cultura y los principios morales de la comunidad.

- La pedagogía tradicional y la pedagogía contemporánea conciben en forma antagónica la forma como se estructura el conocimiento: la pedagogía tradicional concibe el conocimiento como el producto del siguiente orden: pensamiento-operaciónacción. La pedagogia contemporánea concibe esa estructuración bajo un orden contrario: acción-operación-pensamiento.

- La administración educativa que se rige por los cánones marcados por la educación tradicional, deja un margen muy limitado para la estructuración del conocimiento, de acuerdo con las exigencias de la pedagogía contemporánea.

- En consecuencia, las innovaciones pedagógicas han tenido mayor éxito en el campo teórico que en el práctico. Su realización práctica choca contra los parámetros de la administración educativa, que asume la escuela en forma semejante a como lo dejó escrito Comenio en el siglo XVI.

Necesidad de involucrar los valores de una ética cívica en la institución escolar, tanto en el curriculo, como en la evaluación e igualmente en la administración del sistema educativo

El tema concerniente a la necesidad de involucrar los valores de una ética de minimos en la vida institucional se asume a partir del hecho de la urgencia que se requiere de una educación para la autonomía. Ella, como valor de la modernidad, se debe acompañar de los valores que ya se han establecido en una ética civica o ética de mínimos. Alrededor de la vivencia de esos valores, se plantea la posibilidad de 
alcanzar el pleno desarrollo de la personalidad humana y el fortalecimiento de esos valores. Estos -libertad, igualdad, solidaridad-, son los valores guia sobre los que se han construido los derechos humanos y los que permiten un ambiente de pluralidad ideológica. Igualmente es necesario cultivarse en esos otros valores, propios de una ética de minimos, como el respeto activo y el diálogo, sin los cuales los tres primeros no se habrían consolidado, porque los derechos humanos pertenecen a la modernidad, que a su vez exige como valor la autonomía y asume al hombre como un ser con dignidad.

La escuela debe ser el lugar donde se vivan esos valores, pues predicar sobre ellos $\sin$ vivirlos, produce una disyunción entre lo-quedeberia-ser y lo-que-es, que conduce a trasladar esa disyuntiva a todas las otras instituciones de la sociedad, conformadas o dirigidas por los ciudadanos educados en esa dualidad.

Por otro lado, hay que reconocer que las pedagogias contemporáneas han trabajado esos valores, sin embargo, la tradición en cuanto a la administración educativa no hace posible esa vivencia, pues los valores que se han cultivado en la escuela son otros, más vinculados a una concepción premoderna que aún la habita: obediencia, humildad, respeto, templanza.

La administración educativa se vive bajo los parámetros de la pedagogia tradicional, descritos por Comenio en la "Didáctica Magna", que invitan a una ejecución planeada, bajo un orden riguroso, que hace del docente $y$ del directivo docente, un administrador del plan de estudio. El orden comeniano está reforzado en la actualidad, por conceptos gerenciales que hacen del estudiante un ente de conocimiento, no un ser humano con capacidad de conocer.

3. Trilla, como coordinador en una obra denominada El legado pedagógico del siglo XX para la escuela del siglo XXI, en el capítulo 6 , a cargo de Josep M. Puig Rovira, describe algunos ensayos 
pedagógicos que él denomina como antiautoritarios, pretendieron responder al autoritarismo de la educación tradicional. La respuesta estuvo precisamente del lado de los valores de la ética de mínimos, colocando en tela de juicio los valores de la escuela tradicional. Estos ensayos de la pedagogía antiautoritaria son:

- La escuela de Summerhill, con planteamientos donde se resalta la libertad como la base motivacional del deseo de aprender, con argumentos contrapuestos a la forma de trabajo en la educación en general y que son perfectamente analizables para la educación colombiana. Esos planteamientos especificamente son: I. No adoctrinar ni dar instrucciones sobre como comportarse. II. No moldear el carácter. III. No provocar temor ni usarlo para inducir conductas morales. IV. Permitir la autorregulación o capacidad de dirigirse libremente de acuerdo con impulsos que provienen del propio yo. Planteamientos que tal vez se han dado en el imaginario de algún pedagogo pero no hacen parte de la estructura organizacional del sistema educativo. Sólo son apreciables en los marcos teóricos pero, precisamente, no logran trascender.

- La pedagogía no directiva, que apuntó hacia valores como el respeto activo y la solidaridad, que planteó Carl Ramson Rogers, parece ser igualmente imposible en un contexto de imposición dogmática del conocimiento, que es lo que ha imperado en Colombia. Aspectos como los siguientes son los que Rogers argumenta: 1 . Todos los individuos tienen una tendencia innata a actualizar sus posibilidades vitales que se expresa en una capacidad natural de aprender; una capacidad que se activa cuando el sujeto está seguro de si mismo, encuentra un clima de libertad y es valorado positivamente por parte de los educadores. 2. La no directividad es un principio que debe regir todos los intercambios humanos y en especial los educativos, de modo que nadie debe tomar en sus manos los procesos de crecimiento personal y formación de otras personas. Nadie aprende nada relevante bajo presión. 3. El aprendizaje centrado en el alumno supone que no hay desarrollo personal ni adquisición de 
conocimiento si no es a partir de la experiencia de cada sujeto. Nadie debe imponer significados a los demás y nadie aprende nada significativo al margen de sus intereses vitales. 4. Los educadores deben ayudar a los alumnos a desarrollar su impuiso optimizador manifestando actitudes como la empatía, la autenticidad y la confianza. Cuando un alumno se siente personalmente reconocido se incrementa su rendimiento y el valor de lo que aprende.

La pedagogia institucional resalta los valores de la igualdad y el diálogo. Los exponentes de la pedagogía institucional, al igual que Dewey, conciben la escuela como un contexto social y politico. Para ellos la escuela es el agente educativo por excelencia. Esa corriente pretende trabajar en dos niveles simultáneamente: el análisis y la autogestión. Para ellos el error no es condenable, es analizado y sobre él se trabaja. Para el contexto colombiano, sus seguidores podrian estar, tal vez, en los sueh̆os de algunas personas pertenecientes a una comunidad educativa, pero otras realidades dominan la escuela colombiana, donde se ha hecho de la gerencia educativa, con sus nefastos correlatos, en cuanto a excelencia y aprovechamiento, la nueva forma de estratificación social.

La comunidad justa, de Kohlberg, trabaja el valor de la justicia. La comunidad justa no trabaja dilemas de laboratorio. Se trabaja en una comunidad real, por ejemplo el aula. En elia, existen tres instituciones que regulan la vida del colectivo: el grupo asesor, la reunión de la comunidad y la comisión de disciplina. El grupo asesor en el cual hay profesores y estudiantes, prepara la reunión de la comunidad mediante el estudio de las propuestas del orden del día. En la reunión de la comunidad se debaten los problemas y se busca la solución más justa, orientados por criterios de justicia. La comisión de disciplina, formada por profesores y alumnos que participan en forma voluntaria y rotativa, se encarga de mediar en las situaciones de conflicto personal o de clase. Puede adjudicar castigos, cuando asi lo decidan. Con los trabajos con la comunidad 
justa se ha logrado generar un tono antiautoritario en la educación, pero de nuevo en la escuela se imponen otras realidades.

a

Es concluyente que programar la vida de las instituciones educativas alrededor de los valores de una ética de minimos es hasta ahora un reto. Se hace necesario crear un clima institucional en valores como la igualdad, la libertad, la solidaridad, el diálogo, el respeto activo. Igualmente se deben involucrar esos valores, no sólo en el plan de estudio, sino en las actividades que conforman el curriculo, principalmente en los sistemas de administración y valoración de toda la vida institucional.

Por otro lado, la parcelación del tiempo de la escuela en el sistema horario, que provee en forma efectiva el tiempo para desarrollar el plan de estudio, limita enormemente las posibilidades de: 1 . EI intercambio efectivo del conocimiento. 2. El manejo del diálogo como mecanismo de conciliación y evaluación. 3. Consolidar una administración colegiada del centro docente. 4. Permitir que se abra un intercambio real entre la escuela y la comunidad en que está inmersa.

Parece que no sólo es el momento de revisar la efectividad del "mecanismo de relojeria" creado por Comenio, sino de revisar la forma como se trabaja el currículo.

El reto del cambio en las instituciones escolares debe asumirse desde una revisión profunda, crítica y propositiva de la administración, el curriculo, el plan de estudio, los códigos deontológicos de cada una de las instancias, actores e instituciones que hacen posible que la escuela exista, de los aportes que ya se han hecho desde las escuelas pedagógicas, que hablan por si mismas de lo que ha sido funcional y de lo que no ha sido, en cuanto al desarrollo de la personalidad humana. Todo ello para poder involucrar una ética civica en la politica de la escuela, que funciona como un micro mundo social.

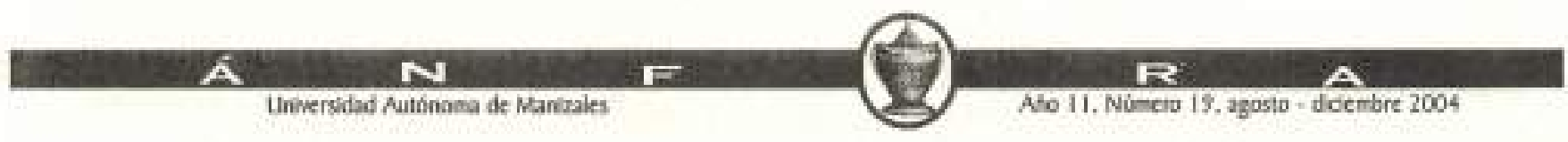




\section{BIBLIOGRAFIA}

CORTINA, Adela. Ética aplicada y demecracia radical. Madrid. Editorial Tecnos S.A. 1997.

MACINTYRE, Alasdair. Tras la virtud. Barcelona. Editorial Crítica. 2002.

MARROU, Henry-Irenee. Historia de la educación en la antigüedad. Madrid: Akal. 1985.

SABINE, George H. Historia de la teoria politica. México: Fondo de cultura económica. 1970.

TRILLA, Jaume. $\mathrm{y}$ otros. El legado pedagógico del siglo $\mathrm{XX}$ para la escuela del siglo XXI.Barcelona: Graó. 10 edición, 2001. 